\title{
The Study on the Design of Low voltage AC Contactor in the Electrical System and the Control Applications
}

\author{
Bao-ying Wang ${ }^{1, a}$ \\ ${ }^{1}$ Chongqing College of Electronic Engineering, Chongqing, 401331, China \\ awangbaoying401331@163.com
}

Keywords: Electrical system; Low voltage AC contactor; Control; Design

\begin{abstract}
The contactor is a kind of main circuit used to frequent connected and cut off and the large capacity control electronics, due to the AC and DC main circuit quickly cut off, and it can frequent switch the large current control circuit, so often be used to control motor, still can be used for machine tools, factory equipment and other electric power load control. Low voltage AC contactor is mainly used for on-off power electric equipment, can remote control the power devices, power on and off equipment to avoid personal injury. The low voltage AC contactor is mainly used for on-off power electric equipment, can remote control the power device, when the on and off equipment the power supply to avoid personal injury. The selection of AC contactor for the normal operation of the power equipment and power lines is Very important. This article puts forward the electrical design process when choosing low voltage AC contactor need to consider the main factors.
\end{abstract}

\section{Introduction}

The contactor can not only control of the circuit to connect and breaking also has the effect of release and protection. Contactor's main contact concatenated in the load circuit, used to load the large current in the circuit connected and breaking, after the contactor coil for electricity excitation, the smaller the excitation current can produce enough to touch the armature magnetic suction, drive the movable contact movement to perform the function of on and off. Its operating frequency in when to open and to cut off the power supply control can reach 1200 times per hour, therefore, AC contactor constantly connected and breaking operations. Due to the AC contactor is the dynamic process of the contactor iron core of the collision and contact bounce of a direct impact, therefore, will inevitably cause of dynamic and static contact between the wear and tear. Because this will produce during the period of high pressure electric arc, the arc ablation of dynamic and static contact will further contact between the wear and tear, and mechanical life of the contactor and electric life an important impact. Especially for use in AC type for AC contactor, when its closed, the contact of the current is 6 times of its rated current, which can be seen, the switching process of AC contactor is the important cause of contact erosion, the reason of its experimental study on process, in the process of AC contactor connected to the main contactor voltage and current waveform, the coil voltage and current waveform for recording, measuring and extract the bounce time and release time and off time, contact performance parameters, to improve the whole performance of AC contactor, predict the system safety hidden dangers, prevent the happening of the accident is of great significance.

\section{The structure and parameters of AC contactor}

The typical structure and principle of AC contactor. AC contactor is on and off the main circuit of contact. Because most of the main circuit of AC contactor is three phase type, so the three pole contact structure is the major structure of the AC contactor contacts. AC contactor's main contact is used to open and close circuit and its auxiliary contact is used to control circuit for conduction. Main contact state usually a normally open state, and the state of the auxiliary contact both the normally open and normally closed state, usually two to often opened two normally closed, AC contactor contact is made by good electrical conductivity and high temperature erosion 
resistance of tungsten alloy made of silver. AC contactor structure includes four parts: 1) composed of static iron core and attract coil electromagnetic system, it is part of the contact with regard to, rely on it to drive the contact closing and opening; 2) consists of three and a pair or two of the main contact for normally open, normally closed auxiliary contact system; 3) Arc extinguishing device, 4) insulation casing and accessories, and Various transmission mechanism, spring, such as short circuit ring and terminal.

The working principle of AC contactor is based on electromagnetic induction, figure 1 as the working principle of AC contactor. Figure 1, 1 is the main contact AC contactor, two auxiliary contact is normally closed, three auxiliary contact is normally open, 4 is moving iron core, 5 is the electromagnetic coil, 6 is the static iron core, 7 is arcing cover, 8 is spring. In the coil after electrify creates a magnetic field, the magnetic field generated electromagnetic suction makes the static iron core 6, the electromagnetic suction makes dynamic core 4 be attracted and downward movement, which makes 1 normally open the main contact closure and auxiliary normally closed contact 2 disconnect, normally open auxiliary contact 3 (usually two) closed. When coil power, produced by the electromagnetic force disappears before, in the spring 8, the dynamic contact reset upwards, each contact to restore the original state.

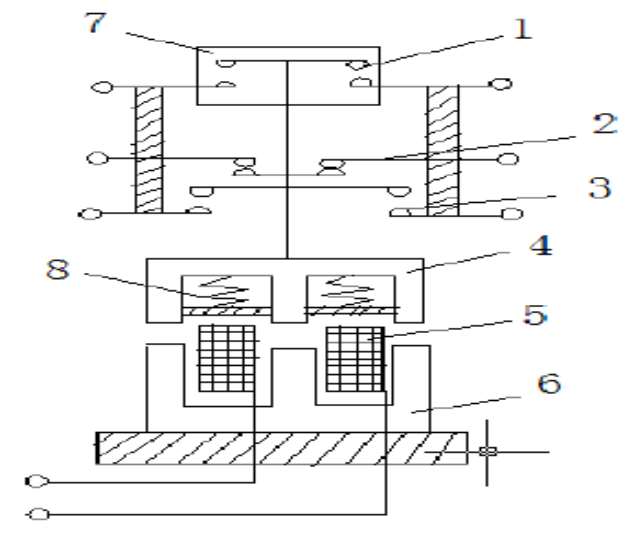

Fig. 1: The working principle of AC contactor

The parameters of the low voltage AC contactor. General use of AC contactor unit structure is compact, easy to use, magnetic blow device for static contact is good, the arc extinguishing effect is good, best achieve zero flashover, temperature rise is small. According to the way of arcing into the air and Vacuum, in accordance with the operation mode can be divided into electromagnetic type, pneumatic type and electromagnetic pneumatic type. Contactor voltage rating parameters are divided into high pressure and low pressure; low pressure generally is $380 \mathrm{~V}, 500 \mathrm{~V}, 660 \mathrm{~V}, 1140 \mathrm{~V}$, etc. It divided into AC and DC current according to the pattern. Current parameters have rated current, conventional thermal current, current is switched on and the breaking current, the provisions of the auxiliary contact heating current and current short-term tolerance of contactor. General contact number shape parameters is given thermal current agreement, agreed fever several corresponding to the rated current. The CJ20-63, for example, the main contact rated current is divided into 63 a, a, 40 model parameters of 63 thermal current refers to the agreement, it and the contactor shell insulation structure, and the rated working current and the selected load current, the voltage level. According to the voltage divided into AC contactor coil 36, 127, 220, 380V, etc. The number of contactor of divided into 2, 3, 4, 5, etc. Auxiliary contacts, according to the normally open normally closed each have a few choice according to the need of control. Other parameters are connected, breaking times, mechanical life, life, maximum allowable operating frequency, the maximum allowable connection wire diameter and shape size and installation size, etc.

For such as contactor, relay switch electrical appliances, it on speed and impact energy produced in the process of closing to a great extent, affect the mechanical and electrical life of its own. In the process of contact action, the occurrence of collision will make contactor contact bounce, in addition, the contactor will be occurred collision between core and static iron core, and it makes contact to produce the second beat. In order to reduce secondary produced by contact bounce, in 
addition to the need to adopt good reaction absorption characteristic cooperate to reduce the energy of the collision, the installation of the buffer device in the electromagnetic system is needed. CJX2-0910 type AC contactor under the static iron core pad some rubber cushion has the effects of the redundant energy absorption core collision. The main factors influencing the reliability of electrical appliances are a dynamic process.

\section{The selection of AC contactor under different load}

The control of electric heating equipment with the selection of AC contactor. Such devices have a resistance furnace, thermal control equipment, etc., the electric heating element used in the winding resistance element, the load current is switched on up to 1.4 times the rated current, if considering the higher power supply voltage, such as the current also get bigger. This kind of load current range is small, by using the categories belong to AC - 1, nor frequent operation, when choosing contactor as long as according to the rated working current of the contactor Ith equal to or greater than 1.2 times working current of electric heating equipment.

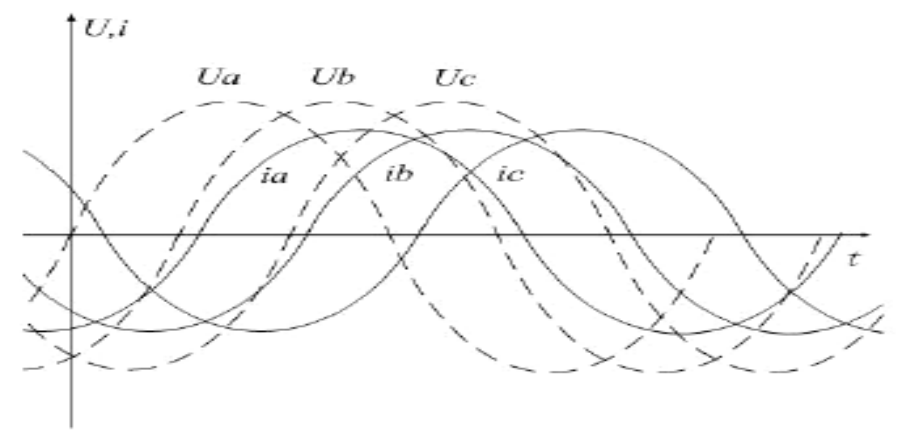

Fig. 2: AC contactor three-phase balance work voltage and current waveform figure

The control of lighting in the selection of the contactor. Many different kinds of lighting equipment, different types of lighting equipment, starting current and start time are different also. Such load use category for AC - 5 a or AC - 5 b. If the start time is very short, can choose the thermal current Ith equal to 1.1 times lighting equipment working current. Start for a long time and low power factor, can choose their heating current Ith than lighting equipment working current. Table 2 is the contactor selection principle for different lighting devices.

The control of arc welding transformer with the selection of contactor. When switching low voltage transformer load, because the transformer secondary side of the electrode short circuit and a short steep and high current, in a larger primary side current, up to $15 \sim 20$ times the rated current, it is associated with the winding and the core characteristics of transformer. When the welder frequently produce sudden strong current, so that the primary side of transformer switch under enormous stress and current, so you must according to the transformer under the rated power of the electrode short circuit when a side of the short circuit current and welding frequency to select the contactor, namely the short circuit current is switched on is greater than the secondary side when the primary side current. Such load is using the categories for AC - 6 a.

The motor with the selection of contactor. Motor with contactor usage according to motor and motor type can choose AC - $2 \sim 4$, respectively, for starting current in 6 times the rated current, rated breaking current of electrical flow can choose AC - 3, such as fan, water pump, we can choose the look-up table method and curve method, according to the sample, and manual selection, need not calculate.

The capacitor contactor is chosen. Capacitor switching capacitor generate transient process, appeared a lot of switching inrush current, accompanied by high frequency oscillation current at the same time, the current from the grid voltage, and the circuit reactance of capacitor capacity decision (i.e., related to the mains transformer and the connection wire), so in the process of contact closure may ablation serious, shall be calculated according to the capacitor circuit in steady state current and practical in the power system on the possible maximum peak flow to choose, which ensures that operate correctly and safely. 
Choose ordinary AC contactor want to consider the flow ratio of switching capacitor Banks, power grid capacity, transformer, circuit, and the impedance of the switchgear, parallel capacitor discharge state, and closing phase Angle, etc., generally rated current up to 50 to 100, when calculation is quite complicated.

Motor for general equipment, working current is less than rated current, although starting current to rated current of $4 \sim 7$ times, but time is short, the contactor contact damage is not big, contactor has been considered in the design of this factor, the general selection contact capacity is greater than the motor rated capacity of 1.25 times. Such as electric hoist to impact load, overload and stop frequently, reverse connect braking and so on, so the working current is multiplied by the corresponding ratio calculation, the overload and stop frequently, use 4 times the motor rated current, reverse connect braking under overload current is usually 2 times starting current, so should choose 8 times the rated current for this condition.

Of AC contactor, disconnected vibration absorption is bigger, try not to more strict and vibration when installation of electrical equipment installed in a cupboard or you'll anti-shock measures, usually installed at the bottom of the cabinet as far as possible. Installed in a sealed environment, avoid dust fall into. Installation environment of AC contactor must conform to the requirements of the product, should conform to the safe distance, electrical wiring installation size discipline, and simple maintenance.

\section{Summary}

The selection of AC contactor is not only relation with the load on and off, and the contactor in the circuit impedance parameters of power system, and also control mode and using the environment and use requirements, so fully consider when choose AC contactor, numerical calculation of parameters, step by step to choose reasonable, and is easy to be used.

\section{References}

[1] Koidl, Hermann P.,Rieder, Werner F.,Salzmann, Quido R.Influence of contact material parameters on contact compatibility of organic vapors. IEEE Transactions on Components and Packaging Technologies. 2010

[2] Kawase, Yoshihiro,Tatsuoka, Satoshi,Yamaguchi, Tadashi,Ito, Shokichi.3-D finite element analysis of operating characteristics of AC electromagnetic contactors. IEEE Transactions on Magnetics. 2013.

[3] Gollee, R.,Gerlach, G.FEM-based method for analysis of the dynamic behavior of AC contactors. IEEE Transactions on Magnetics . 2012.

[4] W Tarczynski,T Hejman,D Smugala.Computer-controlled testing system for investigating the dynamic characteristics of contactors with A.C. electromagnet drives. Measurement : journal of the International Measurement Confederation. 2014. 